\title{
Acrometastasis as the Initial Presentation of a KRAS-Positive Colon Cancer
}

\author{
Helen Gharwan ${ }^{a} \quad$ Lavanya Yarlagadda $^{b} \quad$ Austin Duffy $^{c}$ \\ ${ }^{a}$ Department of Internal Medicine, Johns Hopkins University/Sinai Hospital of \\ Baltimore and ${ }^{b}$ Division of Hematology/Oncology, Department of Medicine, \\ Saint Agnes Hospital, Baltimore, Md., and ' ${ }^{\mathrm{C}}$ Medical Oncology Branch, \\ GI Malignancies Section, National Cancer Institute, National Institutes of \\ Health, Bethesda, Md., USA
}

\section{Key Words}

Colon cancer - Acrometastasis · Finger - Bone - Amputation · KRAS

\begin{abstract}
The propensity of colon cancer to metastasize to bones is very low compared to prostate, breast or lung cancer. The reason for this is not yet understood, although an explanation for the osteotropism of certain primaries has been offered by the 'seed and soil' concept, suggesting that the bone microenvironment provides a favorable 'soil' for metastasis and proliferation of some tumor cells ('seeds') [1]. Here, we report an unusual case of colon cancer with metastasis to the finger at initial presentation, and exophytic sclerotic lesions to other bones. The incidence of metastases to fingers/toes (acrometastases) is reported to be only $0.1 \%$, even after including involvement of primary sites other than the colon. The etiology and characteristics of acrometastases are reviewed in this case report.
\end{abstract}

\section{Case Report}

A 54-year-old African-American male with an unremarkable past medical and family history presented with a gradually worsening and painful swelling of his right middle finger, which severely impaired the use of his hand over several months. On physical examination, a mass was palpated on the proximal phalanx without evidence of fixation to the overlying skin. The nail bed was intact without pigmented lesions or other gross abnormalities. X-ray showed an erosive destructive lesion involving the third metacarpophalangeal joint with adjacent soft tissue swelling and abnormalities of the phalangeal bones adjacent to the joints (fig. 1a; encircled areas). Biopsy and H\&E stain of the metacarpal bone (fig. 1b) and the contiguous cartilage revealed glandular structures, as seen in mucinous adenocarcinoma, suggestive of gastrointestinal or pulmonary origin. Non-contrastenhanced CT scans of the chest, abdomen, and pelvis presented an $8.6 \times 5 \mathrm{~cm}$ dense mass in the ascending colon. No lymph node enlargement was appreciated. Colonoscopy confirmed a large ulcerating obstructing tumor in the ascending colon, and the biopsy result was consistent with 
infiltrating adenocarcinoma. A PET scan showed multiple areas of uptake in the right colon (SUV 8.1), the dome of the liver (SUV 6.3), portocaval and para-aortic lymph nodes (SUV 6.1), and in several bones [right first rib, right side of the sternal manubrium and left posterior tenth rib (SUV 3.7)]. The carcinoembryonic antigen level was elevated at $72.9 \mathrm{ng} / \mathrm{ml}$ (normal $0-3 \mathrm{ng} / \mathrm{ml}$ ).

The patient underwent simultaneous ray amputation of the affected digit and right hemicolectomy. The colonic specimen showed high-grade mucinous adenocarcinoma (fig. 1c; H\&E stain) invading through the muscularis propria into the pericolonic adipose tissue, but not perforating the peritoneal surface. Nine of 24 analyzed lymph nodes contained tumor cells, consistent with the AJCC stage of pT3/N2/M1 (6th edition of AJCC cancer staging handbook). KRAS mutation analysis was positive for a G13D mutation in codon 13 of the KRAS gene.

Chemotherapy with capecitabine $1,000 \mathrm{mg} / \mathrm{m}^{2}$ on days 1 to 14 , oxaliplatin $130 \mathrm{mg} / \mathrm{m}^{2}$ on day 1 , and bevacizumab $7.5 \mathrm{mg} / \mathrm{kg}$ on day 1 of a 21-day cycle was initiated, as was treatment with zoledronic acid every 28 days. Partial remission with resolution of the liver and lymph node lesions was achieved after six cycles. Four months later, an FDG-PET scan revealed increased activity in the sternal manubrium extending into the right first rib (SUV 5.2), corresponding to mixed blastic and lytic changes of an exophytic lesion seen on the CT scan (fig. 2a; arrows). Prominent exophytic changes were also present in the left tenth rib (fig. 2b; arrows), but these did not demonstrate significant glucose uptake. In the abdomen, new focally increased activity was found in the right lobe of the liver (SUV 4.1) and in a right para-aortic lymph node (SUV 4.2). Biopsy of the sternal lesion confirmed KRAS-mutated metastatic adenocarcinoma of the colon. Chemotherapy with capecitabine at $1,000 \mathrm{mg} / \mathrm{m}^{2}$ on days 1 to 14 , irinotecan at $225 / \mathrm{m}^{2}$ on day 1 , and bevacizumab at $7.5 \mathrm{mg} / \mathrm{kg}$ on day 1 of a 21-day cycle was started. Zoledronic acid was continued throughout the treatment. Although the patient initially responded well to this regimen and hepatic abnormalities were no longer detectable after three cycles, a repeat PET/CT scan after six cycles showed that the painless sternal metastasis had grown in size. Diffusely increased activity was noted in both the sternum and the right first rib. Liver involvement continued to remain undetectable, but increased activity was also noted in celiac and peripancreatic lymph nodes. The initial regimen with capecitabine, oxaliplatin, and bevacizumab was resumed, and zoledronic acid was continued. Five months thereafter, the patient developed a painful swelling of the right wrist. X-ray revealed an expansile, partly calcified mass involving the distal right ulna (fig. 2c, d; arrow).

Twenty-three months after initial presentation, no solid organ or lymph node metastases were detectable, while the patient's osseous involvement remained unresponsive to systemic treatment. Palliative radiation was administered to the bone lesions and efforts were made to enroll the patient onto a clinical trial.

\section{Discussion}

Osseous metastases in colorectal cancer (CRC) are rare [2]. They are found in approximately $10 \%$ of all CRC patients [3] - in only $5.5 \%$ of the patients at the time of initial diagnosis [4] - and generally occur in the setting of synchronous metastatic disease at other organ sites [5]. They develop predominantly in the vertebral column, pelvic or hip bones, and the long bones [4]. Radiographically, they are usually osteolytic [4], but an osteoblastic [4] or a mixed osteoblastic-osteolytic appearance [4] with periosteal reactions and calcification of the adjacent soft tissue ('pseudosarcomatous' appearance) is also seen [6].

Metastases to the hands or fingers - so-called acrometastases from the Greek akros, meaning extreme - are very rare, their incidence having been reported to be as low as $0.007-0.2 \%[7,8]$; even after taking into account metastases from primary sites other than the colon. A recent review of the English language medical literature published reported lung, kidney, and breast cancers as the most common origins for phalangeal metastases $(44,12$, and $10 \%$, respectively) [9]. Colon cancer, while ranking low (6\%) as 
the primary tumor site, was found to be the most frequent site of origin among all gastrointestinal tumors. Men are more likely than women to develop finger metastases, and the average age at the time of tumor detection is reported to be 58 years [9]. Interestingly, the right hand appears to be more susceptible to phalangeal metastases, with the middle finger being most frequently affected, followed by the thumb (28 vs. $21 \%$ of all cases analyzed) [9]. Among the bones of the hand, the distal phalanges are the most likely metastatic locations, followed in frequency by the metacarpal bones [9]. Single metastatic finger lesions are more common than multiple lesions.

Partial or ray amputation appears to be the most frequently reported treatment method for acrometastases, although alternatives encompass systemic therapy and radiation, which in the palliative setting can sometimes achieve disease control and alleviation of bony pain. In addition, bisphosphonates were shown to prevent skeletalrelated events in patients with bone metastases from solid tumors [10]; more recently, the human monoclonal antibody denosumab was introduced as a new option to reduce skeletal morbidity in patients with solid tumors that have metastasized to bone [11].

\section{Conclusion}

In summary, this case illustrates a highly unusual manifestation of metastatic CRC involving the fingers. Accurate diagnosis and effective treatment in a timely manner are essential to ensure that patients regain full use of their hands and maintain their independence. While we were able to achieve resolution of the extra-osseous metastases with systemic treatment, the skeletal metastases were refractory to the agents used.

\section{Acknowledgements}

We thank Dr. Albert Aboulafia for patient referral and providing us with the radiographic images, and Drs. Joseph D. Nuckols and Diana W. Molavi for providing us with the histopathology images.

\section{Disclosure Statement}

All authors have no conflict of interest. 

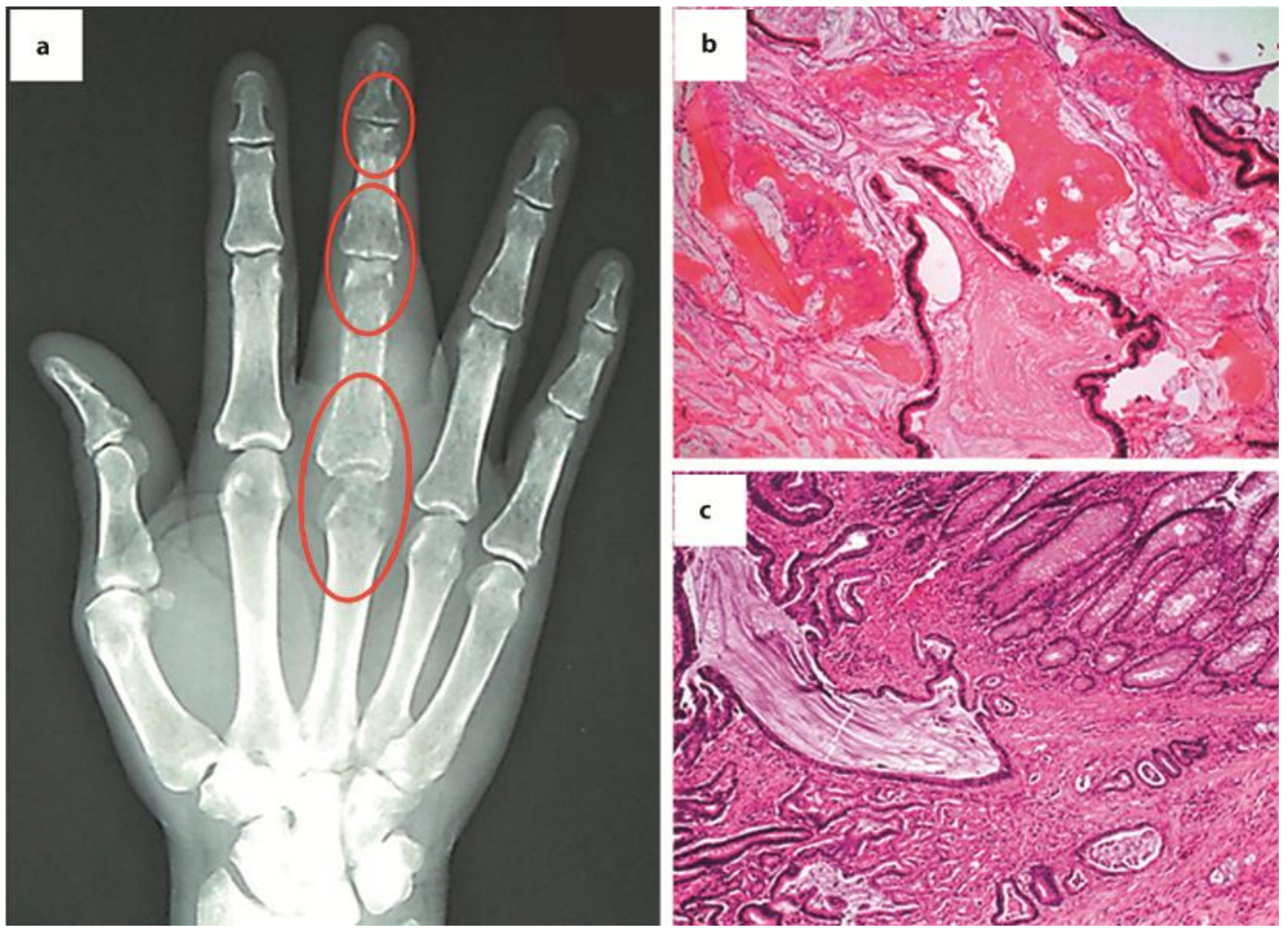

Fig. 1. a X-ray of the patient's right hand, demonstrating an erosive destruction lesion involving the third metacarpophalangeal joint and abnormalities of the phalangeal bones adjacent to the joints (encircled areas). b Biopsy and hematoxylin/eosin stain of the metacarpal bone and the contiguous cartilage revealing glandular structures as seen in mucinous adenocarcinoma. $\mathbf{c}$ The colonic specimen reveals high-grade mucinous adenocarcinoma invading through the muscularis propria into the pericolonic adipose tissue. 

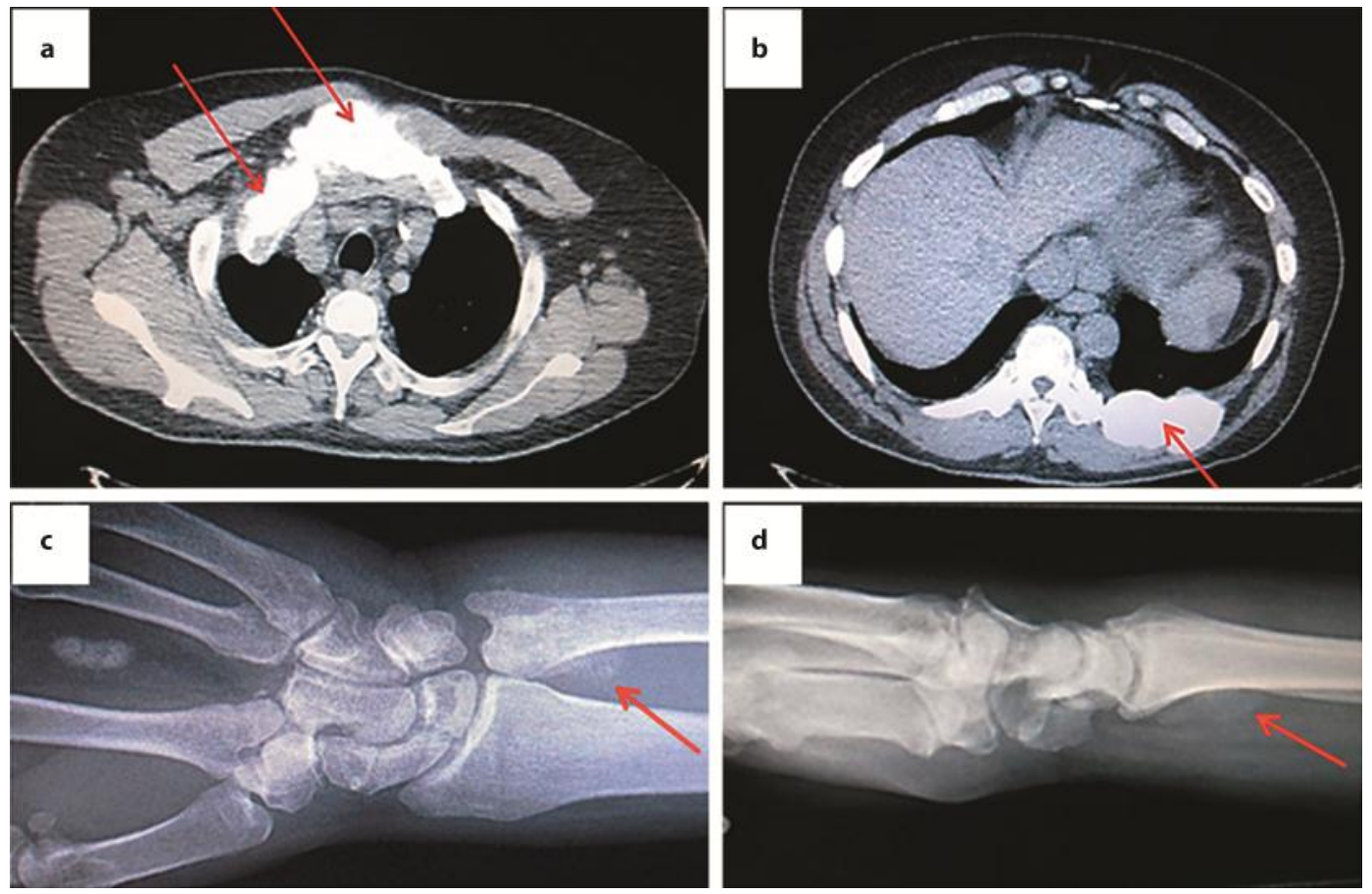

Fig. 2. a CT scan of the chest, demonstrating mixed blastic and lytic changes of an exophytic lesion (arrows). b The arrow points to the prominent exophytic leion of the left tenth rib. c, $\mathbf{d}$ X-ray of the patient's right wrist, demonstrating an expansile, partly calcified mass involving the distal right ulna. c Posteroanterior view; d lateral view.

\section{References}

1 Mundy GR: Mechanisms of bone metastasis. Cancer 1997;80:1546-1556.

- 2 Hess KR, Varadhachary GR, Taylor SH, Wei W, Raber MN, Lenzi R, Abbruzzese JL: Metastatic patterns in adenocarcinoma. Cancer 2006;106:1624-1633.

3 Katoh M, Unakami M, Hara M, Fukuchi S: Bone metastasis for colorectal cancer in autopsy cases. J Gastroenterol 1995;30:615-618.

4 Santini D, Tampellini M, Vincenzi B, Ibrahim T, Ortega C, Virzi V, Silvestris N, Berardi R, Masini C, Calipari N, Ottaviani D, Catalano V, Badalamenti G, Giannicola R, Fabbri F, Venditti O, Fratto ME, Mazzara C, Latiano TP, Bertolini F, Petrelli F, Ottone A, Caroti C, Salvatore L, Falcone A, Giordani P, Addeo R, Aglietta M, Cascinu S, Barni S, Maiello E, Tonini G: Natural history of bone metastasis in colorectal cancer: final results of a large Italian bone metastases study. Ann Oncol 2012, Epub ahead of print.

$\checkmark 5$ Roth ES, Fetzer DT, Barron BJ, Joseph UA, Gayed IW, Wan DQ: Does colon cancer ever metastasize to bone first? A temporal analysis of colorectal cancer progression. BMC Cancer 2009;9:274-279.

6 Oh YK, Park HC, Kim YS: Atypical bone metastasis and radiation changes in a colon cancer: a case report and a review of the literature. Jpn J Clin Oncol 2001;31:168-171.

-7 Anoop TM, George S, Divya KP, Jabbar PK: Metastatic phalangeal osteolysis as an initial presentation of carcinoma colon. Am J Surg 2010;200:e61-e63.

8 Sur YJ, Kang YK, Bahk WJ, Chang DK, Rhee SK: Metastatic malignant tumour in the hand. J Plast Surg Hand Surg 2011;45:90-95.

-9 Flynn CJ, Danjoux C, Wong J, Christakis M, Rubenstein J, Yee A, Yip D, Chow E: Two cases of acrometastasis to the hands and review of the literature. Curr Oncol 2008;15:51-58.

10 Ibrahim A, Scher N, Williams G, Sridhara R, Li N, Chen G, Leighton J, Booth B, Gobburu JV, Rahman A, Hsieh Y, Wood R, Vause D, Pazdur R: Approval summary for zoledronic acid for treatment of multiple myeloma and cancer bone metastases. Clin Cancer Res 2003;9:2394-2399.

11 Saylor PJ: Targeted therapies: Denosumab - a new option for solid tumors metastatic to bone. Nat Rev Clin Oncol 2011;8:322-324. 